\title{
219 CD47 AND PHOSPHATIDYLSERINE CONTRIBUTE TO THE INTERACTION BETWEEN ANTIGEN PRESENTING CELLS AND THE ALLOGENEIC CELL-BASED RELAPSE VACCINE DCP-001
}

${ }^{1}$ Haoxiao Zuo, 'Satwinder Kaur Singh*, 'Marie-José Van Lierop, 'Jorn Kaspers, 'Remco Bos, ${ }^{2}$ Alwin Kamermans, ${ }^{2}$ Helga E de Vries, ${ }^{2}$ Tanja de Gruijl, ${ }^{1}$ Ada Kruisbeek, 'Erik Manting, ${ }^{1}$ Satwinder Kaur Singh. ${ }^{1}$ Immunicum $A B$, Leiden, Netherlands; ${ }^{2}$ Amsterdam University Medical Center, Amsterdam, Netherlands

Background DCP-001 is a cancer relapse vaccine derived from the DCOne ${ }^{\circledR}$ human leukemic cell line. During manufacturing, DCOne $^{\circledR}$ cells are shifted towards a mature dendritic cell $(\mathrm{mDC})$ phenotype, combining an endogenous tumor antigen repertoire (e.g. WT-1, RHAMM and PRAME) with a mDC costimulatory profile and providing the basis for the highly immunogenic vaccine DCP-001. In a phase I clinical study in acute myeloid leukemia (AML), DCP-001 demonstrated to be safe and to induce multifunctional antitumor immune responses. ${ }^{1}$ It has also been reported that DCP-001 induces antitumor immunity against multiple myeloma cells in peripheral blood mononuclear cells (PBMC) from multiple myeloma patients and that DCP-001 antigenic material is transferred to host antigen presenting cells (APC), possibly via extracellular vesicles. $^{2}$ However, the possibility of direct interactions between DCP-001 and host APC has not yet been investigated.

Methods To further elucidate the mode of action of DCP-001, we studied the interactions of DCP-001 with human PBMC and isolated immature monocyte-derived DCs (iMoDC) in in vitro co-culture studies. A human skin explant model was used to determine uptake of DCP-001 by migrating skin DCs after intradermal injection.

Results We found that DCP-001 stimulates the secretion of various proinflammatory cytokines (IL-1 $\beta$, GM-CSF, IFN- $\gamma$, IL2 , TNF- $\alpha$, and IL-6) and chemokines (IL-8 and RANTES) in PBMC. In addition, we demonstrate that DCP-001 is efficiently taken up by iMoDC via direct cell-cell interactions and that this phagocytic process is influenced by "eat-me" and "don't eat me" signaling pathways. Blocking of the "eat-me" signals calreticulin and phosphatidylserine inhibited the uptake of DCP-001, whereas blockade of the "don't eat me" signal CD47 enhanced DCP-001 uptake. After intradermal injection of DCP-001 in an ex-vivo human skin model, its uptake by skin-emigrating DCs was demonstrated as well as simultaneous activation of these DCs.

Conclusions Our data suggest a key role for host antigen presenting cells in the triggering of immune responses upon DCP-001 vaccination. In addition, the data provide rationale for potential combination therapies based on DCP-001 and inhibitors of the CD47 pathway.

\section{REFERENCES}

1.. van de Loosdrecht AA, et al. A novel allogeneic off-the-shelf dendritic cell vaccine for post-remission treatment of elderly patients with acute myeloid leukemia. Cancer Immunol Immunother 2018:67(10):1505-1518.

2.. Leaf RK, et al. DCOne as an allogeneic cell-based vaccine for multiple myeloma. J Immunother 2017:40(9):315-322.

http://dx.doi.org/10.1136/jitc-2021-SITC2021.219 